\title{
Arzthaftungsmodul der FMH und SIM für medizinische Gutachter
}

\section{Iris Herzog-Zwitter}

Dr. iur., Abteilung Rechtsdienst FMH/SIM-Bildungsbeauftragte

\author{
Die FMH hat gemeinsam mit der Swiss Insurance Medicine (SIM) ein Arzthaftungs- \\ modul für medizinische Gutachterinnen und Gutachter entwickelt. Diese neue \\ ärztliche Fortbildung erhöht die Qualität der Gutachten bei Arzthaftungsverfahren \\ und fördert den interdisziplinären Wissensaustausch. Das Modul wird am 2. De- \\ zember 2021 auf Deutsch und am 1. Juli 2021 auf Französisch angeboten.
}

Medizinerinnen und Mediziner, die sich in Arzthaftungsgutachten spezialisieren bzw. das Fachwissen zum Arzthaftungsrecht vertiefen wollen, finden im neuen Arzthaftungsmodul von FMH und SIM ein massgeschneidertes Angebot. Das interdisziplinäre Zusammenwirken und das gegenseitige Verständnis von Medizin und Recht sind wichtige Elemente für das

Experten aus Medizin und Rechtswissenschaft vermitteln Fachwissen anhand von konkreten Fällen und juristischem Basiswissen.

Erstellen eines Gutachtens im Arzthaftungsrecht. Damit ein solches Dokument den rechtlichen Anforderungen entspricht, müssen die Verfassenden neben dem medizinischen Fachwissen der entsprechenden Spezialität auch über das juristische Fachwissen und die rechtswissenschaftliche Terminologie verfügen.

\section{Lernen am konkreten Fall}

Nachdem das Arzthaftungsmodul in Rahmen eines Pilotprojekts im Januar 2019 am Unispital Basel erfolgreich erprobt worden ist, hat im August 2020 in der Klinik Hirslanden in Zürich die erste Durchführung stattgefunden. Die referierenden Expertinnen und Experten aus Medizin und Rechtswissenschaft vermitteln Fachwissen anhand von konkreten Fällen und juristischem Basiswissen. Im deutschsprachigen Arzthaftungsmodul werden die medizinischen Fachdisziplinen Orthopädie und Gynäkologie im Zentrum stehen, jedoch auch weitere medizinische Fachbereiche zur Sprache kommen.

\section{Von der Sorgfaltspflicht bis zur Telemedizin}

Das Arzthaftungsmodul greift nicht nur die Kernthemen der ärztlichen Sorgfaltspflicht, der Aufklärungspflicht und der Dokumentationspflicht auf, sondern widmet sich auch dem Thema der Kommunikation bei einem vermuteten Behandlungsfehler. Ferner wird das Gemeinschaftliche Gutachterkonsilium ein innovatives Verfahren der Begutachtung - näher ausgeführt. Weiter stehen Themen wie Fehler und "No-Gos» in den Formulierungen, Brennpunkte der medizinischen Notfallversorgung sowie aktuelle haftpflichtrechtliche Fragestellungen im Kontext der Telemedizin auf dem Programm.

\section{Arzthaftungsmodul:}

\section{Termine und Anmeldung}

Das nächste Arzthaftungsmodul von FMH und SIM in deutscher Sprache wird am 2. Dezember 2021 von 15 bis 21.30 Uhr in der Klinik Hirslanden in Zürich durchgeführt. Auf Französisch findet die Fortbildung am 1. Juli 2021 von 14 bis 20.30 Uhr in Lausanne statt oder per Videoübertragung.

In der Kursgebühr von 500 Franken sind Teilnahmebestätigung und Verpflegung inbegriffen. Das SIWF und die SIM vergeben je 6 Credits für dieTeilnahme am Arzthaftungsmodul. Weitere Informationen zum Arzthaftungsmodul sowie das Anmeldungsformular finden Sie unter:

www.fmh.ch/gutachterausbildung-arzthaftung 